\title{
SPECTRAL RADIUS FORMULAE
}

\author{
by G. J. MURPHY and T. T. WEST
}

(Received 13th June 1978)

\section{Introduction}

If $A$ is a complex Banach algebra (not necessarily unital) and $x \in A, \sigma(x)$ and $r(x)$ will denote the spectrum and spectral radius of $x$ in $A$. If $I$ is a closed two-sided ideal in $A$ let $x+I$ denote the coset in the quotient algebra $A / I$ containing $x$. Then

$$
r(x+I) \leqslant \inf _{y \in I} r(x+y)
$$

Smyth and West (7) have considered algebras for which equality holds in formula (*) for each $x \in A$ and each closed two-sided ideal $I$. We call such algebras $S R$-algebras. They showed that the following familiar algebras (i) $C_{0}(\Omega)$ where $\Omega$ is a locally compact Hausdorff space (ii) $L^{\prime}(G)$ where $G$ is a locally compact abelian group and (iii) $B(X)$ where $X$ is $c_{0}$ or $l_{p}(1 \leqslant p<\infty)$ are SR-algebras, while the algebra of analytic functions on the unit disc in the supremum norm fails to be $S R$.

If $A$ is commutative with Gelfand space $\Sigma(A)$ and with Gelfand transform algebra $\hat{A}$ we prove in Proposition 1 that $A$ is SR if $\hat{A}$ is dense in $C_{0}(\Sigma(A))$. A partial converse to this result in Proposition 2 states that if $A$ is regular, commutative and $S R$, then $\hat{A}$ is dense in $C_{0}(\Sigma(A))$.

It was conjectured in (7) that every $C^{*}$-algebra is SR. This was proved by Pedersen in (3). A quick proof of this theorem is a consequence of the following formula for the spectral radius of any element $x$ in a unital $C^{*}$-algebra $A$ given in Proposition 4,

$$
r(x)=\inf _{a=a^{*} \in A}\left\|e^{a} x e^{-a}\right\| \text {. }
$$

The proof consists of showing first that $r(x)=$ inf $\left\|y x y^{-1}\right\|$ over the invertible elements in $A$, and then taking the logarithm of the positive part of the polar decomposition of $y$. These formulae appear to be new for general $C^{*}$-algebras.

A trivial observation that the Riesz algebras introduced by Smyth in (6) are SR leads to the fact that a large class of Banach algebras are SR.

\section{Commutative algebras}

Let $A$ be a commutative Banach algebra. If $x \in A$ we denote by $\hat{x}$ the Gelfand transform of $x$. $\hat{A}$ is the Gelfand transform algebra of $A$ and if $\Sigma(A)$ is the Gelfand space, then $\hat{A} \subseteq C_{0}(\Sigma(A))$. $A$ is symmetric if $\hat{A}$ is closed under complex conjugation. If $I$ is an 
ideal in $A$ (always taken to be closed), $h(I)$ denotes the hull of $I$. If $f \in C_{0}(\Sigma(A))$ and $\Omega \subseteq \Sigma(A)$ we write $|f|_{\Omega}$ for the supremum of $f$ on $\Omega$. Thus if $x \in A$,

and since $\Sigma(A / I)=h(I)$

$$
r(x)=|\hat{x}|_{\Sigma(A)}
$$

$$
r(x+I)=|\hat{x}|_{h(I)}
$$

Proposition 1. Let $A$ be a commutative Banach algebra such that $\hat{A}$ is dense in $C_{0}(\Sigma(A))$. Then $A$ is an SR-algebra.

Proof. Let $J$ denote the closure of $\hat{I}$ in $C_{0}(\Sigma(A))$. Then, by hypothesis, $J$ is an ideal in $C_{0}(\Sigma(A))$ also $h(J)=h(I)$. Thus, since $C_{0}(\Sigma(A))$ is SR,

$$
\begin{aligned}
r(x+I) & =|\hat{x}|_{h(I)}=|\hat{x}|_{h(J)}=\inf _{f \in J}|\hat{x}+f|_{\Sigma(A)} \\
& =\inf _{y \in I}|\hat{x}+\hat{y}|_{\Sigma(A)}=\inf _{y \in I} r(x+y) .
\end{aligned}
$$

Corollary. If $A$ is symmetric or if $\Sigma(A)$ is totally disconnected then $A$ is an SR-algebra.

Proof. If $A$ is symmetric the Stone-Weierstrass theorem implies that $\hat{A}$ is dense in $C_{0}(\Sigma(A))$. If $\Sigma(A)$ is totally disconnected this is also true (8, Corollary 8 .)

This improves the result in (7) Proposition 1.

The converse problem of characterising commutative Banach algebras which are $\mathrm{SR}$ is not completely solved, but we have the following result.

Proposition 2. Let $A$ be a regular commutative algebra which is $S R$. Then $\hat{A}$ is dense in $C_{0}(\Sigma(A))$.

Proof. Let $B$ be the closure of $\hat{A}$ in $C_{0}(\Sigma(A) . B$ separates the points of $\Sigma(A)$ thus $\Sigma(B)=\Sigma(A)$.

If $\Omega$ is a compact subset of $\Sigma(A)$ put $I=k(\Omega)$ the kernel of $\Omega$ in $A$. Then $h(I)=\Omega$ by regularity. Let $J$ be the closure of $\hat{I}$ in $B$. Then $J$ is an ideal of $B$ and $h(J)=h(I)=\Omega$. Now if $x \in A$

$$
\begin{aligned}
\|\hat{x}+J\| & =\inf _{f \in J}\|\hat{x}+f\|=\inf _{y \in I}\|\hat{x}+\hat{y}\|=\inf _{y \in I} r(x+y) \\
& =r(x+I) \quad \text { (by hypothesis) } \\
& =|\hat{x}|_{\Omega} .
\end{aligned}
$$

Thus, since $\hat{A}$ is dense in $B$,

$$
\|f+J\|=|f|_{\Omega} \quad(f \in B)
$$


Denoting by $B \mid \Omega$ the restriction algebra of $B$ to $\Omega$ we have shown that $B / J$ is isometric to $B \mid \Omega$. Therefore $B \mid \Omega$ is closed in $C(\Omega)$ for each compact subset $\Omega$ of $\Sigma(A)$. It follows from (8) Corollary 13.9 that $B=C_{0}(\Sigma(A))$.

\section{3. $\mathrm{C}^{*}$-algebras}

If $A$ is a unital $C^{*}$-algebra, $\operatorname{Inv}(A)$ denotes the set of invertible elements of $A$.

Lemma. Let $x$ be an element of a unital $C^{*}$-algebra $A$ such that $r(x)<1$. Then there exists $y \in \operatorname{Inv}(A)$ such that $\left\|y x y^{-1}\right\|<1$.

Proof. Since $r(x)<1$ the series $\sum_{n=0}^{\infty}\left\|\left(x^{n}\right)^{*} x^{n}\right\|=\sum_{n=0}^{\infty}\left\|x^{n}\right\|^{2}$ is convergent. Thus $z=\sum_{n=0}^{\infty}\left(x^{n}\right)^{*} x^{n} \in A$ and $z \geqslant 1$. Therefore $y=z^{1 / 2} \in A$ and $y \geqslant 1$ so $y \in \operatorname{Inv}(A)$. Moreover

$$
\begin{aligned}
\left\|y x y^{-1}\right\|^{2} & =\left\|y^{-1} x^{*} y^{2} x y^{-1}\right\| \\
& =\left\|y^{-1} \sum_{n=1}^{\infty}\left(x^{*}\right)^{n} x^{n} y^{-1}\right\| \\
& =\left\|1-y^{-2}\right\| \\
& =r\left(1-y^{-2}\right) \\
& <1 .
\end{aligned}
$$

Therefore $\left\|y x y^{-1}\right\|<1$ as required.

Proposition 4. If $A$ is a unital $C^{*}$-algebra

$$
r(x)=\inf _{a=a^{*} \in A}\left\|e^{a} x e^{-a}\right\|=\inf _{y \in \operatorname{Inv}(A)}\left\|y x y^{-1}\right\| \quad(x \in A) .
$$

Proof. If $x \in A$ choose a positive number $\delta$ such that $r\left(x \delta^{-1}\right)<1$. By Lemma 3 there exists $y \in \operatorname{Inv}(A)$ such that $\left\|y x \delta^{-1} y^{-1}\right\|<1$. As $y$ is invertible, it has a polar decomposition $y=u|y|$ in $A$, where $u$ is a unitary element of $A$ and $|y|=\left(y^{*} y\right)^{\frac{1}{2}}$. Thus $|y| \in \operatorname{Inv}(A)$ and there exists $\epsilon>0$ such that $\sigma(|y|) \subseteq(\epsilon, \infty)$. Hence $a=\log (y) \in A$, and $a$ is hermitian. Also

$$
\left\|y x y^{-1}\right\|=\left\|u e^{a} x e^{-a} u^{*}\right\|=\left\|e^{a} x e^{-a}\right\|<\delta,
$$

as $u$ is unitary.

Hence

$$
\inf _{a=a^{*} \in A}\left\|e^{a} x e^{-a}\right\| \leqslant r(x)
$$

and since for each $a \in A$

$$
r(x)=r\left(e^{a} x e^{-a}\right) \leqslant\left\|e^{a} x e^{-a}\right\|
$$

the reverse inequality holds, and the theorem is proved. 
Proposition 4 leads to a short proof of the fact that every $\mathrm{C}^{*}$-algebra is SR.

Corollary. $C^{*}$-algebras are $S R$-algebras.

Proof. If $I$ is a closed two-sided ideal of a non-unital $C^{*}$-algebra $A$, then $I$ is also a closed two-sided ideal of the $\mathrm{C}^{*}$-algebra formed by adjoining an identity. Thus it is sufficient to prove the result for unital $C^{*}$-algebras $A$. By Proposition 4 if $x \in A$, since $A / I$ is a $C^{*}$-algebra

$$
\begin{aligned}
r(x+I) & =\inf _{a \in A}\left\|e^{a+I}(x+I) e^{-(a+I)}\right\| \\
& =\inf _{a \in A}\left\|e^{a} x e^{-a}+I\right\| \\
& =\inf _{a \in A} \inf _{y \in I}\left\|e^{a}(x+y) e^{-a}\right\| \\
& =\inf _{y \in I} \inf _{a \in A}\left\|e^{a}(x+y) e^{-a}\right\| \\
& =\inf _{y \in I} r(x+y) .
\end{aligned}
$$

We record that Rota (4) proved the second part of the formula in Proposition 4 in the case $A=B(H)$.

\section{Riesz algebras}

Let $A$ be a complex Banach algebra with $\operatorname{radical} \operatorname{Rad}(A)$. If $A^{\prime}=A / \operatorname{Rad} A$, the socle of $A^{\prime}, \operatorname{Soc}\left(A^{\prime}\right)$, exists. $A$ is called a Riesz algebra by Smyth (6) if the hull of the pre-image of Soc $A^{\prime}$ is empty, in other words the pre-image of Soc $A^{\prime}$ is contained in no primitive ideal of $A$. Smyth (8, Theorem 10$)$ has proved that a Banach algebra is a Riesz algebra if, and only if, the non-zero part of the spectrum of every element is a countable discrete set.

Proposition 5. A Banach algebra which is a Riesz algebra is $S R$.

Proof. Let $A$ be such an algebra and let $I$ be a closed two-sided ideal in $A$. If $x \in A$ let $0 \neq \lambda \in \sigma(x)$, The spectral projection $p=p(\lambda ; x)$ associated with the isolated point $\lambda \in \sigma(x)$ is an element of $A(5$, lemma 2.1). Clearly $p+I$ is the spectral projection in $A / I$ associated with the isolated point $\lambda \in \sigma(x+I)$. Thus

$$
\lambda \in \sigma(x+I) \Leftrightarrow p+I \neq I \Leftrightarrow p \not I .
$$

Now if $\lambda \notin \sigma(x+I), p x \in I$ and $\lambda \notin \sigma(x-p x)$.

Smyth's characterisation implies that the set $\{\mu \in \sigma(x):|\mu|>t>0\}$ is a finite set. Denote the associated spectral projection by $p_{t}$. Then

$$
r(x+I) \leqslant t \Leftrightarrow p_{t} \in I \Rightarrow p_{t} x \in I .
$$


since $r\left(x-p_{t} x\right) \leqslant t$ this gives

$$
r(x+I) \leqslant t \Rightarrow \inf _{y \in I} r(x+y) \leqslant t,
$$

whence the result.

In the semi-simple case the Riesz algebras introduced by Smyth are exactly the modular annihilator algebras of Barnes (1). Consequently the following Banach algebras are Riesz algebras: algebras of compact operators, inessential operators or Riesz operators, algebras with a dense socle, semi-simple dual algebras, semi-simple annihilator algebras, compact algebras and completely continuous algebras. Since closed subalgebras and quotient algebras of Riesz algebras are again Riesz algebras, it follows that closed subalgebras and quotient algebras formed from the above class of algebras are also SR.

\section{REFERENCES}

(1) B. A. BARNES, Examples of modular annihilator algebras, Rocky Mountain J. Math. 1 (1971), 657-663.

(2) J. DIXMIER, Les $C^{*}$-algebres et leurs representations (Gauthier-Villars, Paris, 1964).

(3) G. K. PEDERSEN, Spectral formulas in quotient C*-algebras, Math. Zeit. 148 (1976), 299-300.

(4) G. C. RotA, On models for linear operators, Comm. Pure Appl. Math. 13 (1960), 469-472.

(5) M. R. F. SMYTH, Riesz theory in Banach algebras, Math. Zeit. 145 (1975), 145-155.

(6) M. R. F. SMYTH, Riesz algebras, Proc. Roy. Irish Acad. 76(A) (1975), 327-333.

(7) M. R. F. SMYTH, and T. T. WEST, The spectral radius formula in quotient algebras, Math. Zeit. 145 (1975), 157-161.

(8) E. L. STouT, The theory of uniform algebras (Bogden and Quigley, Tarrytown-onHudson, 1971).

SCHOOL OF Mathematics

Trinity College

Dublin 